\title{
1D goes 2D: A Kosterlitz Thouless transition in superconducting arrays of 4-Angstrom carbon nanotubes
}

\author{
Zhe Wang ${ }^{1}$, Wu Shi ${ }^{1}$, Hang Xie ${ }^{1}$, Ting Zhang ${ }^{1}$, Ning Wang ${ }^{1}$, Zikang Tang ${ }^{1}$, \\ Xixiang Zhang ${ }^{1,2}$, Rolf Lortz ${ }^{1}$, and Ping Sheng ${ }^{1 *}$
}

We report superconducting resistive transition characteristics for array(s) of coupled 4Angstrom single wall carbon nanotubes embedded in aluminophosphate-five (AFI) zeolite. The transition was observed to initiate at $15 \mathrm{~K}$ with a slow resistance decrease switching to a sharp, order of magnitude drop between 7.5-6.0K. The transition has strong (anisotropic) magnetic field dependence. Differential resistance versus current (voltage) measurements indicate that the establishment of coherence proceeds in stages as the temperature is lowered below $15 \mathrm{~K}$. In particular, the sharp resistance drop and its attendant nonlinear IV characteristics are consistent with the manifestations of a Kosterlitz-Thouless (KT) transition that establishes quasi long range order in the plane transverse to the c-axis of the nanotubes, leading to an inhomogeneous system comprising 3D superconducting regions connected by weak links. Global coherence is established at below $5 \mathrm{~K}$ with the appearance of a well-defined supercurrent gap at $2 \mathrm{~K}$.

$\mathrm{S}$ uperconductivity in carbon nanotubes has been a controversial issue because carbon is not known to be a superconducting element, and if there is indeed superconducting tendency in carbon nanotubes ${ }^{1}$, its manifestation could be quenched by long wavelength thermal fluctuations as well as by the Peierls distortion that favors an insulating ground state. In this context the earlier report on the Meissner effect in 4-Angstrom carbon nanotube-zeolite composites $^{2}$ and the more recent observation of their superconducting specific heat signals $^{3}$ have only deepened the mystery on the specific manner in which the nanotube superconductivity comes into being, and on whether there can be a sharp superconducting resistive transition that is usually taken to be the hallmark of a superconductor. In this work we show that by devising strategy to make surface electrical contacts to the samples that are separated by only $100 \mathrm{~nm}$, reliable and repeatable observations of the superconducting resistive transition can be obtained. The overall physical picture that emerges is that of a coupled Josephson array consisting of aligned nanotubes crossing over from an individually fluctuating $1 \mathrm{D}$ system to a coherent 3D superconductor, mediated by a Kosterlitz-Thouless (KT) transition ${ }^{4}$ which establishes quasi long range order in the lateral plane perpendicular to the c-axis of

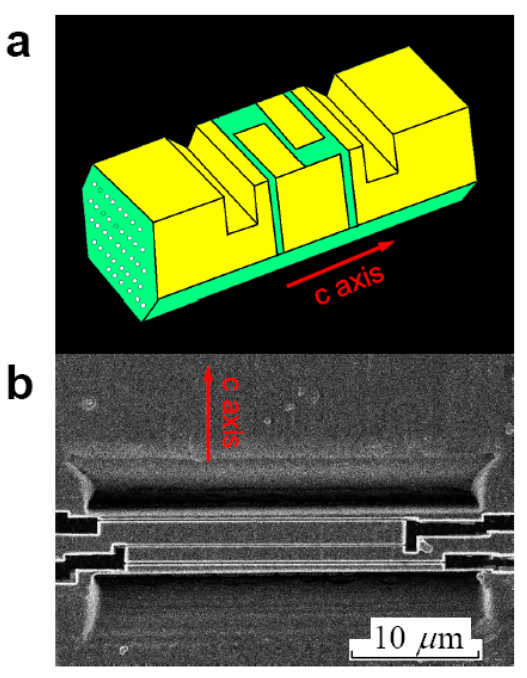

Figure 1 | Geometry of the electrical contacts.

a, Cartoon picture of AFI zeolite crystal with the 4-probe contact geometry. Yellow denotes gold and green denotes AFI crystal surface exposed by FIB etching. Nanotubes, denoted schematically by open circles, are aligned along the $c$ axis.

b, A SEM image of the sample with the contact geometry illustrated in $\mathbf{a}$. The dark areas are the deep troughs. The $100 \mathrm{~nm}$-width line etched by the FIB is visible in the center of the image. It separates the two surface electrodes.

\footnotetext{
${ }^{1}$ Department of Physics and William Mong Institute of Nano Science and Technology, HKUST, Clear Water Bay, Kowloon, Hong Kong, China.

${ }^{2}$ Present address: Research and Development, King Abdullah University of Science and Technology, Thuwal, Saudi Arabia.

*Corresponding author. Email address: sheng@ust.hk.
} 
the nanotubes. The attainment of overall coherence across the measuring electrodes (denoted as global coherence in this work) is seen at $5 \mathrm{~K}$ and below, accompanied by the appearance of a well-defined (differential resistance) supercurrent gap at $2 \mathrm{~K}$.

\section{Electrical contact geometry}

Figure 1 shows both a cartoon picture (1a) of the AFI zeolite crystal with the 4-probe contact geometry, as well as a scanning electron microscope (SEM) image of an actual sample (1b). Here the crystal was prepared by first cutting two troughs in an AFI crystal $(50 \times 50 \times 500 \mu \mathrm{m})$ with focused ion beam (FIB, Seiko SMI2050). The troughs are separated by a $5 \mu \mathrm{m}$ slice that is perpendicular to the c-axis (Fig. 1a).

The straight pores of the AFI zeolite are aligned along the c-axis with a center-to-center separation of $1.37 \mathrm{~nm}$. They form a 2D close-packed triangular lattice in the plane transverse to the c-axis. The inner diameter of the pores, after discounting the size of the oxygen atoms lining the walls, is $0.7 \mathrm{~nm}^{5}$. The 4 Angstrom nanotubes are embedded in the AFI zeolite pores. There can be three types of 4-Angstrom carbon nanotubes that are consistent with optical and Raman data $^{6-10}:(5,0),(4,2)$ and $(3,3)$. We attribute the superconducting behavior to the $(5,0)$ nanotubes.

The AFI zeolite crystal with embedded nanotubes was sputtered with $50 \mathrm{~nm}$ of Ti and 150nm of Au. The electrical contact geometry was subsequently delineated by using the FIB to remove the $\mathrm{Au} / \mathrm{Ti}$ film in a pre-designed pattern, shown in Fig. 1b. Here the outer electrodes make end contacts to the nanotubes, whereas the two inner electrodes, each about $2 \mu \mathrm{m}$ wide, are separated by $100 \mathrm{~nm}$ and are on the surface of the AFI crystal. As the nanotubes are only $\sim 1 \mathrm{~nm}$ below the surface, which is imperfect in any case, the surface contact electrodes enables the measurement of electrical characteristics transverse to the c-axis of the nanotubes, i.e., the contact resistance in this case is the transverse resistance. We have carried out measurements using both the four-probe (with the outer contacts as the current electrodes) and the twoprobe geometry. In the latter the two surface-contact electrodes were used. In what follows we show results mostly done in the two-probe geometry because of the importance of the transverse resistance in the KT transition mechanism. Use of four-probe geometry is noted whenever such result is presented,

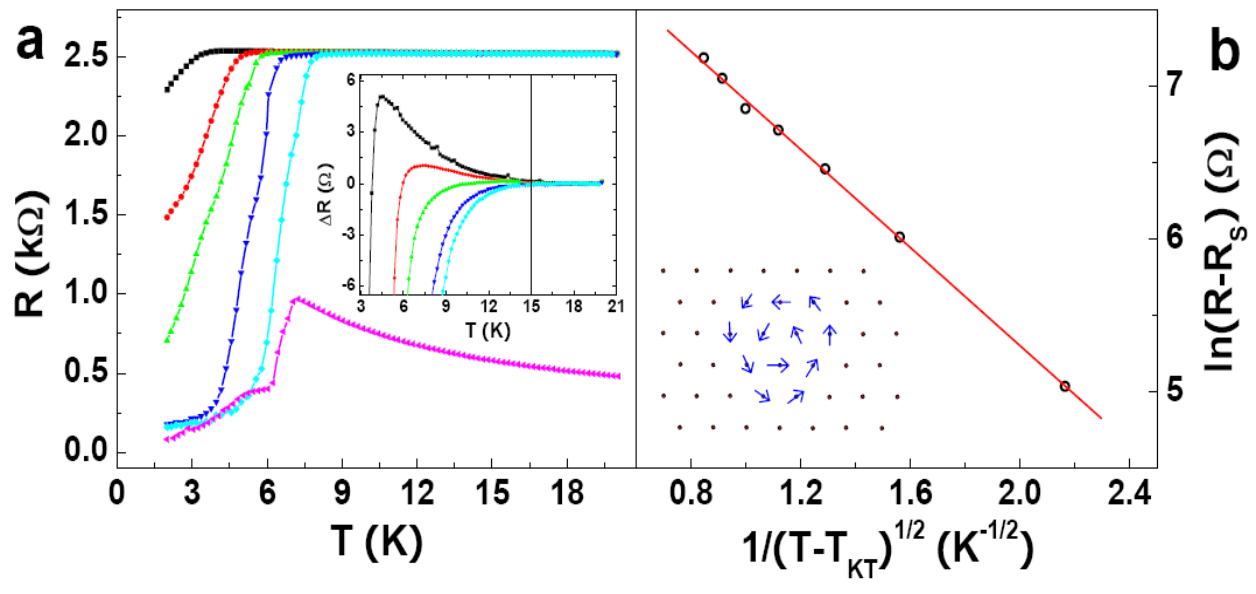

Figure 2 | Temperature dependence of resistance measured with a magnetic field applied perpendicular to the c axis.

a, Resistance measured at $1 \mu \mathrm{A}$ plotted as a function of temperature. At zero field, the sharp drop initiates at $\sim 7.5 \mathrm{~K}$. The transition is pushed to lower temperatures with applied magnetic field, but still not completely quenches at $9 \mathrm{~T}$. This sharp drop in resistance is attributed to a KT transition in the plane perpendicular to the $c$ axis. The magenta curve is the four leads data at zero field, with the other curves measured with the two probe geometry. From right to left, the light blue curve denotes $0 \mathrm{~T}$, dark blue for $1 \mathrm{~T}$, green for $3 \mathrm{~T}$, red for $5 \mathrm{~T}$, and black for $9 \mathrm{~T}$. The same color coding applies to the curves in the inset. The nonmetallic temperature dependence of the four-probe result at $T>7.5 \mathrm{~K}$ is indicative of the discontinuities of the nanotubes, leading to hopping conduction and weak links. Inset: A magnified view of the upper section of the resistance vs. temperature curves from $3 \mathrm{~K}$ to $20 \mathrm{~K}$ with the straight-line asymptote above $17 \mathrm{~K}$ subtracted. The superconducting transition clearly begins at $15 \mathrm{~K}$.

b, Under zero field, the $R(T)$ associated with a KT transition is predicted to behave as $\ln \left(R-R_{S}\right) \propto\left(T-T_{K T}\right)^{-1 / 2}$ for $T>T_{K T}$, denoted by the solid red line. Very good agreement is seen with the data, denoted by open circles. Here $T_{K T}=6.17 \mathrm{~K}$ and $R_{S}=1.06 \mathrm{k} \Omega$ is the series resistance associated with the lower plateau value in Fig. 4 . It arises from the weak links connecting the different superconducting regions at low temperatures. Inset: A schematic picture of the lateral plane perpendicular to the c-axis, in which the KT transition occurs. Here each dot represents an end-view of a segment of the 1D element with a constant phase. The vortex is indicated by closed rings of arrows, representing unit vectors whose directions are given by the phase (angles) of the $1 \mathrm{D}$ element. 
mainly to delineate the electrical anisotropy through comparison with the two-probe results.

\section{Temperature and magnetic field dependences of resistance}

In Fig. 2 we plot the resistance measured as a function of temperature. The most prominent feature is the sharp drop starting at $\sim 7.5 \mathrm{~K}$, which moves to lower temperatures with applied magnetic field (perpendicular to the c-axis in this case). The fourprobe result, measured at zero field, is also displayed for comparison. While the sharp drop feature is preserved in the four-probe data, there is also a kink at $6 \mathrm{~K}$, with a small plateau extending about half a degree below that. In the inset to Fig. 2a we show an enlarged upper section of the curves from 3-20K. For $T>17 \mathrm{~K}$ the curves are very good straight lines with a slight negative slope; the data shown in the inset are measured relative to this straight-line asymptote, extended to lower temperatures.

There are several notable features of the data. First, the fact that the transition is sensitive to the magnetic field, in the range of $1 \mathrm{~T}$ or above, means that the superconducting behavior must originate from a fairly extensive array of coupled nanotubes at least a few tens of nanometers in its lateral size ${ }^{11}$. Similar behavior as displayed in Fig. 2a has been observed in three different samples, with the same drop at $7.5 \mathrm{~K}$, although the absolute values of the measured resistances are different. Second, the inset clearly shows the initiation of the whole transition process starts at $15 \mathrm{~K}$. While the resistance changes are small, their magnetic field dependence is unmistakable. Third, the 4-probe data shows that there could be two stages in reaching global coherence. This point is reinforced by the IV characteristics as shown below. In Fig. $2 b$ we show the zero field resistance versus temperature data to be in excellent agreement with the theoretical prediction based on our interpretation of the $7.5 \mathrm{~K}$ transition as a KT transition with a . This is a key point that will be further elaborated, together with the nonlinear IV characteristics below $7.5 \mathrm{~K}$ that are consistent with the manifestations of the KT transition.

In Fig. 3a we show the magnetoresistance (MR) of the same sample at different temperatures. Here the magnetic field is applied perpendicular to the c-axis. At $2 \mathrm{~K}$, there is a kink at $\sim 2 \mathrm{~T}$. For comparison, fourprobe data are also shown. It is instructive that the measured resistances for the two sets of data are almost identical below $2 \mathrm{~T}$, but diverge above that, i.e., the contact resistance of the surface electrodes can be turned on and off through either temperature or magnetic field. It implies that at $T=2 \mathrm{~K}$ and field below $2 \mathrm{~T}$ the transverse (contact) resistance is markedly decreased, apparently due to increased 3D coherence $^{12}$. Figure $3 \mathrm{~b}$ shows the observed magnetoresistance anisotropy in a separate but similar sample measured at 5T, the maximum in terms of the observed anisotropy. The anisotropy means that a magnetic field perpendicular to the c-axis is more effective in suppressing the superconducting behavior than the same field applied parallel to the c-axis. This is reasonable since the magnetic susceptibility should be larger for the perpendicular field, owing to the fact that it would be easier to induce diamagnetic current loops that are dominantly along the c-axis of the nanotubes, as compared to those that are in the plane transverse to the c-axis.

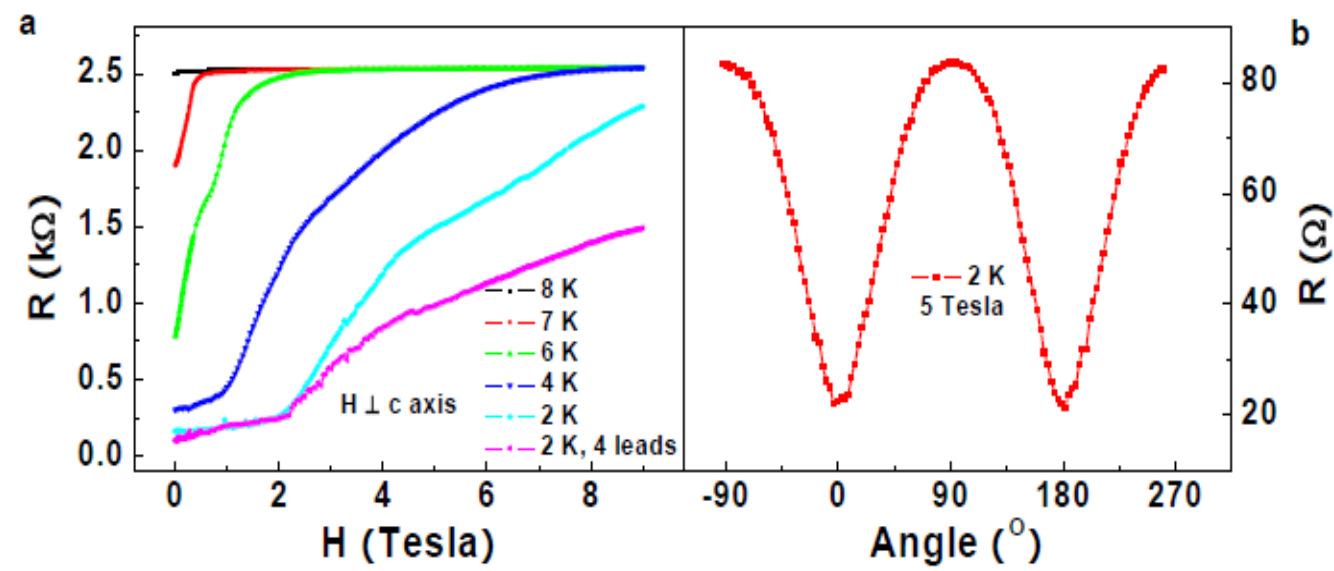

Figure 3 | Magnetoresistance and its anisotropy.

a, Magnetoresistance (MR) measured at $1 \mu \mathrm{A}$ for field applied perpendicular to the $\mathrm{c}$ axis. At $2 \mathrm{~K}$, there is a clear transition at $\sim 2.1 \mathrm{~T}$ for both the two-probe and four-probe data. The two sets of data are about the same magnitude below this field value but diverge above that. This is consistent with the physical picture that global coherence is established at $2 \mathrm{~K}$, and a field above $2 \mathrm{~T}$ would gradually destroy lateral coherence (see Fig. $4 \mathrm{~b}$, the green curve).

b, MR anisotropy measured on another sample (with 100nA current) that displays qualitatively similar behavior as shown in Fig. 2 and Fig. 3a. Here the angle is between the magnetic field and the $c$ axis. 


\section{Physical interpretation}

Our nanotube arrays may be considered as composed of 1D superconducting elements which can be either single nanotubes or small bundles of nanotubes each with strong lateral coherence. We characterize each 1D element with a complex Ginzburg-Landau ${ }^{13}$ wave function $\psi$ which is a function of the spatial variable $x$ along the c-axis. At temperatures above $7.5 \mathrm{~K}$ the $1 \mathrm{D}$ superconducting condensate can experience strong thermal fluctuations in the form of phase slips, leading to finite resistance. Neighboring elements are coupled via Josephson interaction energy $-J \cos \left(\varphi_{i}-\varphi_{j}\right)$, where

$\varphi_{i}$ denotes the phase of the wavefunction for the $i$ th element (see inset to Fig. 2b), assumed to be constant over a finite segment of length $d$, and $J$ is proportional to the paired (zero temperature) superconducting electron density $|\psi|^{2}$. We approximate the system as quasi $2 \mathrm{D}$ in the lateral plane perpendicular to the c-axis with a plane (film) thickness $d$. In such an approximation the Josephson coupling is equivalent to a $2 \mathrm{D}$ spin model with the interaction between neighboring spins expressible as $-J\left(\vec{s}_{i} \cdot \vec{s}_{j}\right)$, where $\vec{s}$ denotes a unit vector in the lateral plane.

It is well known that there can be vortex excitations in $2 \mathrm{D}$ spin systems, consisting of spins that form a closed loop. Vortices of opposite helicities interact as $2 \mathrm{D}$ charges and tend to bind together with a logarithmic potential ${ }^{14,15}$. They can be separated at a certain transition temperature, $T_{K T} \approx 2.8 \mathrm{~J} / k_{B}$, denoted the Kosterlitz-Thouless (KT) transition. As (unpaired) moving vortices can destroy coherence in the lateral plane, its resistance would increase sharply at the KT transition. The appearance of KT transition is noted to be not restricted to thin film superconducting systems; more recently it was also observed in bulk $3 \mathrm{D}$ high $\mathrm{T}_{\mathrm{c}}$ superconductors that are composed of $2 \mathrm{D}$ superconducting atomic layers. These include KT transition observed for vortex excitations within the superconducting layers ${ }^{16-18}$, as well as for interlayer vortex excitations involving multiple superconducting layers $^{19-21}$.

In our experiments, the surface contact electrodes in the two-probe geometry can effectively detect the lateral resistance change ${ }^{12}$, more so than the fourprobe geometry. Comparison between the two sets of data shows that above the KT transition the sample's resistance is more anisotropic (see Fig. 2)-whereas the measured two-probe and four-probe resistances are almost the same below $6 \mathrm{~K}$ (indicating 3D isotropy), they diverge above that.

We attribute the large resistance drop seen at $7.5 \mathrm{~K}$ (Fig. 2a) to result from a KT transition that establishes quasi long range order in the lateral plane ${ }^{22}$, leading to the quench of longitudinal fluctuations and the formation of $3 \mathrm{D}$ coherent regions connected by weak links. Global coherence is attained at even lower temperatures.

It is known that the KT transition, which involves only a small entropy change, occurs at a temperature below the main specific heat peak ${ }^{23-26}$ that reflects the growth of the transverse coherence. Hence the KT transition attribution is consistent with the previous specific heat results, obtained with a 5T quenching field $^{3}$. In this case, however, there could be synergistic effect between the longitudinal and transverse coherence manifest in the specific heat signal at $T>7.5 \mathrm{~K}$, that is absent in the standard model of the KT transition. The specific heat signal associated with the KT transition is yet to be observed; it may require better sample quality than that attained in the past, measured with a higher quenching field.

For the transverse-plane KT transition, the effect of the magnetic field is mainly due to its influence on $J$. A perpendicular magnetic field has the effect of suppressing superconductivity as seen in Fig. 3a. In particular, the superconducting electron density and hence the Josephson coupling energy $J$ are reduced. That has the effect of both shifting $T_{K T}$ to lower temperatures as well as diminishing the magnitude of the resistance drop associated with the transition. A parallel field will have a similar effect, although less effective than the perpendicular field as seen in Fig. $3 \mathrm{~b}$, owing to the smaller magnetic susceptibility (and hence a larger magnetic penetration length).

\section{KT transition characteristics}

In Fig. 4 we show measured differential resistance plotted as a function of the current. The most striking feature is the low temperature differential resistance gap/quasigap centered at zero bias, which disappears in stages with increasing temperature (4a) or applied magnetic field (4b). A notable aspect of the data is the existence of two resistance plateaus at the large current limit, one at $1 \mathrm{k} \Omega$ and the other at $2.3 \mathrm{k} \Omega$. The latter is associated with the $\mathrm{KT}$ transition at $7.5 \mathrm{~K}$. A particularly instructive curve in Fig. $4 \mathrm{a}$ is the one at $6 \mathrm{~K}$ (blue), which shows that the bottom of the KT transition's quasigap coincides with the $1 \mathrm{k} \Omega$ plateau. This is consistent with the physical picture that the $1 \mathrm{k} \Omega$ resistance is associated with the weak links connecting the $3 \mathrm{D}$ superconducting regions. It also justifies treating the $1 \mathrm{k} \Omega$ plateau as the reference from which the KT transition's lateral plane resistance is to be measured.

In the presence of series resistance internal to the sample, differential resistance measurement can help to clarify the intrinsic nature of the $7.5 \mathrm{~K}$ transition. As the temperature is lowered below $7.5 \mathrm{~K}$, it is seen from Fig. 4a that a triangular-shaped quasigap develops which eventually merges into the $2.3 \mathrm{k} \Omega$ plateau with increasing current. The resistance in the $I \rightarrow 0$ limit, measured relative to the $1 \mathrm{k} \Omega$ plateau, is predicted to vary with temperature as ${ }^{27,28}$ 


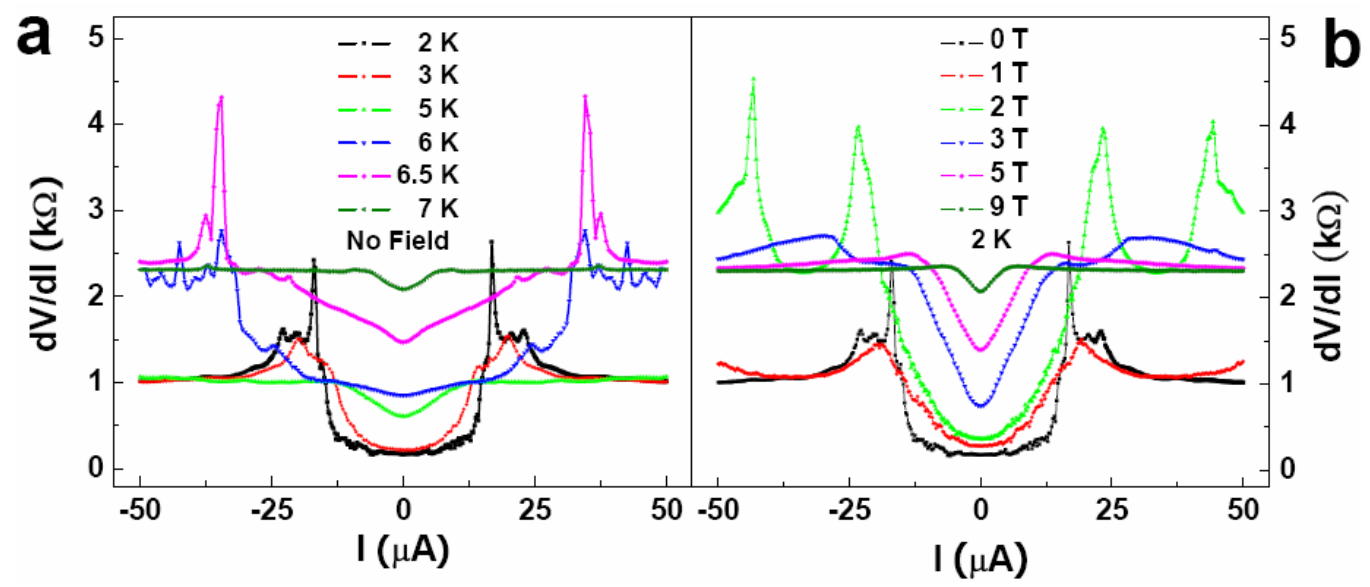

Figure 4 | Current dependence of the differential resistance.

a, Temperature dependence of the differential resistance plotted as a function of the measuring current. At $2 \mathrm{~K}$, the flat platform centered at $I=0$ is a clear manifestation of the supercurrent gap. It disappears in stages when temperature increases and the global coherence is broken. The $1 \mathrm{k} \Omega$ plateau is associated with weak links that connect the $3 \mathrm{D}$ superconducting regions. Above $6 \mathrm{~K}$, lateral coherence deteriorates, and the shape of the quasigap is a reflection of the KT transition's nonlinear $I-V$ behavior at $T<T_{c 0}$. The triangular-shaped quasigap is noted to be associated with the 2.3 $\mathrm{k} \Omega$ plateau.

b, Differential resistance at $2 \mathrm{~K}$ under different applied (perpendicular) magnetic fields. The effect of the magnetic field is qualitatively similar to that shown in $\mathbf{a}$, but differs in details. At $3 \mathrm{~T}$ and above the appearance of the triangular quasigap signals the suppression of the KT transition to below $2 \mathrm{~K}$.

$R-R_{s}=10.8 b R_{n} \exp \left\{-2\left[b\left(T_{c 0}-T_{K T}\right) /\left(T-T_{K T}\right)\right]^{1 / 2}\right\}$ for $T>T_{K T}$. Here $R_{s}=1.06 \mathrm{k} \Omega$ is the lower plateau resistance and $T_{c 0}=7.5 \mathrm{~K}$ is the mean field transition temperature below which there can be nonlinear IV characteristics. In Fig. $2 b$ we show our data to be in excellent agreement with the above prediction. The parameter values obtained are $T_{K T}=6.17 \mathrm{~K}, \quad b=0.48$, and $R_{n}=0.96 \mathrm{k} \Omega$ for the normal sheet resistance. As the measuring current $I$ increases, we find that at $T<T_{c 0}=7.5 \mathrm{~K}$ there is a distinctive regime where the differential resistance $R$ varies linearly as a function of $I$, implying $V \propto I^{2}$. The progressive variation from a constant $R(V \propto I)$ at $7.5 \mathrm{~K}$ to a triangular quasigap $\left(V \propto I^{2}\right)$ and then to a more rounded gap at below $6.5 \mathrm{~K}$, is consistent with the $\mathrm{KT}$ transition behavior of $V \propto I^{\alpha}$ with an $\alpha$ varying from 1 (at the mean field transition temperature $T_{c o}$ ) to 3 (at $T_{K T}$ ) or larger with decreasing temperature ${ }^{23,29}$. From our differential resistance data $\alpha=3$ occurs at $6 K<T<6.5 K$, which agrees well with our earlier estimate of $T_{K T}=6.17 \mathrm{~K}$.

At $6 \mathrm{~K}$ and below, a smaller gap is seen to develop which is associated with the $1 \mathrm{k} \Omega$ plateau. At $2 \mathrm{~K}$, a supercurrent gap becomes well-defined, attributable to the establishment of global coherence. The sharp peaks at the boundaries of the supercurrent gap arise from the existence of a critical current density at which voltage first appears (when the weak links are overcome). This general behavior is seen to be repeated in Fig. 4b, in which the applied magnetic field may be viewed as having the effect of decreasing the transition temperature. In particular, the switching of the plateau resistance at $2 \mathrm{~T}$, from $1 \mathrm{k} \Omega$ at below $2 \mathrm{~T}$ to $2.3 \mathrm{k} \Omega$ at $2 \mathrm{~T}$, is noted to be also reflected in the behavior shown in Fig. 3a, where the curve associated with $2 \mathrm{~K}$ has a kink at $\sim 2 \mathrm{~T}$, beyond which the MR increases with a much larger slope. From the appearance of the triangular quasigap at magnetic fields above $2 \mathrm{~T}$, we conclude that a $3 \mathrm{~T}$ field is sufficient to suppress $T_{K T}$ to below $2 \mathrm{~K}$, but $T_{c 0}$ can remain above $2 \mathrm{~K}$ even at $9 \mathrm{~T}$. An overall phase diagram is presented in the Supplementary section. It should be noted that the peaks seen in Fig. $4 b$ for the $2 \mathrm{~T}$ curve (in light green) remain unexplained. Further studies are required to clarify their origin(s).

\section{Concluding remarks}

We have shown that by measuring 4-Angstrom carbon nanotubes embedded in AFI zeolite at close electrode separations the superconducting behavior of coupled nanotube arrays can be clearly delineated through resistance versus temperature, resistance versus magnetic field, and I-V characteristics. The necessity of using close electrode separation, and the related consequence of small room temperature resistance of the samples (in which the superconducting behavior is manifest), indicate that impurities and defects present in most conducting nanotube samples may be responsible for masking their intrinsic characteristics at the scale of $0.5 \mu \mathrm{m}$ or larger. This is reasonable as impurities and defects are much more effective in localizing electrons in 1D than they are in higher dimensions. 
While superconductivity in carbon nanotubes is now beyond reasonable doubt, results presented in this work also open up some intriguing questions requiring further investigations, among them the fundamental issue of the pairing mechanism in carbon nanotubes. Research on these problems represents theoretical and experimental challenges to be further pursued.

\section{Methods}

The 4 Angstrom carbon nanotube-zeolite composites were prepared by first heating the crystals in 0.3 atmosphere of oxygen and 0.7 atmosphere of $\mathrm{N}_{2}$ or $\mathrm{Ar}$ at $580^{\circ} \mathrm{C}$ for 4 hours. The purpose of this initial heating stage is to remove the precursortripropylamine - that was present in the pores of the as-made crystals. At the end of the first heating stage the crystals were transparent with no Raman signals for carbon-carbon bonds. Subsequently the 0.3 atmosphere of $\mathrm{O}_{2}$ was replaced by ethylene and the crystals were heated at the same temperature for the same duration. The resulting crystals show strong optical polarization anisotropy with Raman radial breathing modes at both $510 \mathrm{~cm}^{-1}$ (for the $(4,2)$ ) and $550 \mathrm{~cm}^{-1}$ (for the $(5,0)$ ). In particular, the peak at the $550 \mathrm{~cm}^{-1}$ is about $10 \%$ the height of the G-band at $1600 \mathrm{~cm}^{-1}$ for the $\mathrm{C}-\mathrm{C}$ bonds ${ }^{2}$.

The transport measurements were carried out in the Quantum Design PPMS, with a $2.1 \Omega$ series resistance. Both resistance and differential resistance were measured using Keithley 2182A Nanovoltmeter and $6221 \mathrm{AC} / \mathrm{DC}$ current source.

\section{References and footnotes}

1. Benedict, L. X., Crespi, V. H., Louie, S. G., \& Cohen, M.. L., Static conductivity and superconductivity of carbon nanotubes: Relations between tubes and sheets, Phys Rev B 52, 1493514940 (1995).

2. Tang, Z. K., Zhang, L. Z., Wang, N., Zhang, X. X., Wen, G. H., Li, G. D., Wang, J. N., Chan, C. T. \& Sheng, P., Superconductivity in 4-Angstrom single-walled carbon nanotubes. Science 292, 2462-2465 (2001).

3. Lortz, R., Zhang, Q. C., Shi, W., Ye, J. T., Qiu, C. Y., Wang, Z. He, H. T., Sheng, P., Qian, T. Z., Tang, Z. K., Wang, N., Zhang, X. X., Wang, J. N. \& Chan, C. T., Superconducting characteristics of $4-\AA ̊$ carbon nanotube-zeolite composite. PNAS 106, 7299-7303 (2009).

4. Kosterlitz, J. M. \& Thouless, D. J., Ordering, metastability and phase transitions in two-dimensional systems. J. Phys. C: Solid State Phys. 6, 1181-1203 (1973).

5. Meier, W. M. \& Olson, D. H., Atlas of zeolite structure types. Zeolites 12, 1-195 (1992).

6. Wang, N., Tang, Z. K., Li, G. D. \& Chen, J. S., Single-walled 4Å carbon nanotube arrays. Nature 408, 50-51 (2000).

7. Tang, Z. K., Sun, H. D., Wang, J., Chen, J. \& Li, G., Mono-sized single-wall carbon nanotubes formed in channels of $\mathrm{AlPO}_{4}-5$ single crystal. Appl. Phys. Lett. 73, 2287-2289 (1998).

8. Li, I. L., Li, G. D., Liu, H. J., Chan, C. T. \&Tang, Z. K., Chirality-dependent curvature effect in smallest single-walled carbon nanotubes. Appl. Phys. Lett. 82, 1467-1469 (2003).

9. Li, Z. M., Liu, H. J., Ye, J. T., Chan, C. T. \& Tang, Z. K., Optical properties of 0.4-nm single-wall carbon nanotubes aligned in channels of $\mathrm{AlPO}_{4}-5$ single crystals. Appl. Phys. A 78, 11211128 (2004).

10. Liu, H. J. \& Chan, C. T., Properties of $4 \AA$ carbon nanotubes from first-principles calculations. Phys. Rev. B 66, 115416 (2002).
11. This sample differs from the one whose electrical resistance vs. temperature was shown in ref. 3 , which reflects the behavior of a 1D (fluctuating) superconducting bundle that presumably acts as the critical link bridging the two surface electrodes.

12. Whereas in the four probe measurements the voltage reflects mainly the current flow through the c-axis direction (since the voltage measurement diverts only a small fraction of the current), in the two probe measurements using the surface electrodes the resistance is mainly from the transverse direction.

13. Tinkham, M., Introduction to Superconductivity. Ch. $4\left(2^{\mathrm{n}}\right.$ edit., MGH, New York, 1996).

14. Doniach, S. \& Huberman, B. A., Topological excitations in twodimensional superconductors. Phys. Rev. Lett. 42, 1169-1172 (1979).

15. Beasley, M. R., Mooij, J. E. \& Orlando, T. P., Possibility of vortex-antivortex pair dissociation in two-dimensional superconductors. Phys. Rev. Lett. 42, 1165-1168 (1979).

16. Martin, S., Fiory, A. T., Fleming, R. M., Espinosa, G. P. \& Cooper, A. S., Vortex-pair excitation near the superconducting transition of $\mathrm{Bi}_{2} \mathrm{Sr}_{2} \mathrm{CaCu}_{2} \mathrm{O}_{8}$ crystals. Phys. Rev. Lett. 62, $677-$ 680 (1989).

17. Bulaevskii, L. N., Ledvij, M. \& Kogan, V. G., Fluctuations of vortices in layered high- $\mathrm{T}_{\mathrm{c}}$ superconductors. Phys. Rev. Lett. 68, 3773-3776 (1992).

18. Matsuda, Y., Komiyama, S., Onogi, T., Terashima, T., Shimura, K. \& Bando, Y., Thickness dependence of the KosterlitzThouless transition in ultrathin $\mathrm{YBa}_{2} \mathrm{Cu}_{3} \mathrm{O}_{7-\delta}$ films. Phys. Rev. B 48, 10498-10503 (1993).

19. Chattopadhyay, B. \& Shenoy, S. R., Kosterlitz-Thouless signatures from 3D vortex loops in layered superconductors. Phys. Rev. Lett. 72, 400-403 (1994).

20. Sugano, R., Onogi, T. \& Murayama, Y., Nonlinear currentvoltage characteristics of three-dimensional Josephson-junction arrays with anisotropic interactions. Phys. Rev. B 48, 1378413788 (1993).

21. Pradhan, A. K., Hazell, S. J., Hodby, J. W., Chen, C., Hu, Y. \& Wanklyn, B. M., Observation of the Kosterlitz-Thouless transition and of vortex fluctuations in superconducting single crystals of Bi-based cuprates. Phys. Rev. B 47, 11374-11378 (1993).

22. While it is possible to have vortex excitations in the plane parallel to the c-axis of the nanotubes, we ascribe the KT transition to be associated with vortices in the plane perpendicular to the c-axis because the relatively constant $7.5 \mathrm{~K}$ transition temperature implies a single $J$ value, which can only be specified through the lattice structure of the zeolite pores. However, as the occupation fraction for the superconducting nanotubes in the lateral plane must be less than $100 \%$, determined by the projection of the overlapping (along the caxis) nanotubes onto the lateral plane, it remains an open possibility that the transition temperature could be dependent on the fraction ${ }^{30,31}$ of superconducting nanotubes in the zeolite pores.

23. Gupta, R. \& Baillie, C. F., Critical behavior of the twodimensional XY model. Phys. Rev. B 45, 2883-2898 (1992).

24. Ding, H. Q. \& Makivic, M. S., Kosterlitz-Thouless transition in the two-dimensional quantum XY model. Phys. Rev. B 42, 6827 6830 (1990).

25. Gupta, R., DeLapp, J. \& Batrouni, G. G., Phase transition in the 2D XY model. Phys. Rev. Lett. 61, 1996-1999 (1988).

26. Tobochnik, J. \& Chester, G. V., Monte Carlo study of the planar spin model. Phys. Rev. B 20, 3761-3769 (1979).

27. Halperin, B. I. \& Nelson, D. R., Resistive transition in superconducting films. J. Low Temp. Phys. 36, 599-616 (1979).

28. Abraham, D. W., Lobb, C. J., Tinkham, M. \& Klapwijk, T. M., Resistive transition in two-dimensional arrays of superconducting weak links. Phys. Rev. B 26, 5268-5271 (1982).

29. Hebard, A. F. \& Fiory, A. T., Critical-exponent measurement of a two-dimensional superconductor. Phys. Rev. Lett. 20, 16031606 (1983)

30. Zeng, X. C., Stroud, D., Chung, J. S., Monte Carlo studies of percolation in a superconducting array. Phys. Rev. Lett.B 43, 3042-47 (1991). 
31. Yun, Y.-J., Baek, I.-C., Choi, M.-Y., Phase transition and critical dynamics in site-diluted Josephson-junction arrays. Phys. Rev. Lett. 97, 215701 (2006).

\section{Acknowledgements}

We thank Bei Zhang, Fuyi Jiang and Milky Tang for technical support. P.S. thanks Qiucen Zhang for helpful discussions. This work was supported by the Research Grants Council of Hong Kong Grants HKUST9/CRF/08, CA04/04.SC02, plus DSC104/05.SC01 and VPAAO04/05.SC01.

\section{Supplementary information}

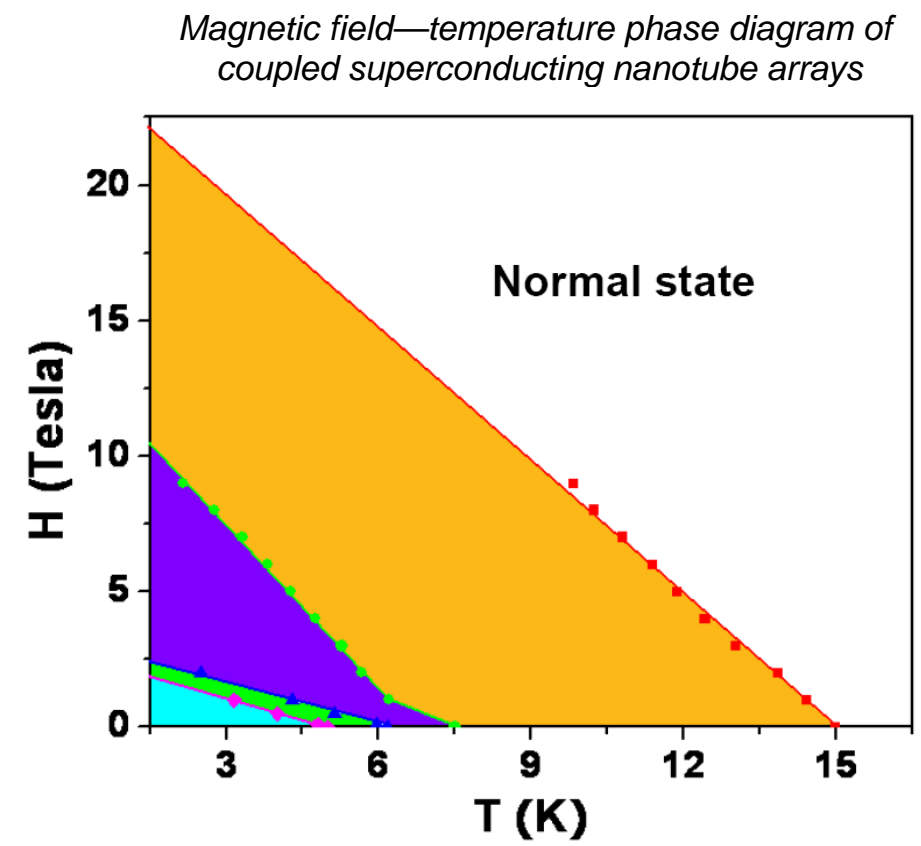

In the phase diagram above, yellow denotes the 1D fluctuating superconductor regime, the green line denotes $T_{c 0}$ and the blue line denotes $T_{K T}$ associated with the KT transition. Area colored by violet is the regime where one expects to see nonlinear IV characteristics, owing to vortex excitations. Green is the regime in which the sample is characterized by inhomogeneous 3D superconducting regions connected by (normal) weak links. The bottom left corner is the regime of global coherence. Here symbols are data; straight line extrapolations are used to delineate the different regimes. 